\title{
Hyperspectral Reconstruction in Biomedical Imaging Using Terahertz Systems
}

\author{
Zhimin $\mathrm{Xu}$ and Edmund Y. Lam \\ Imaging Systems Laboratory, Department of Electrical and Electronic Engineering, \\ The University of Hong Kong, Pokfulam Road, Hong Kong \\ Email: \{zmxu,elam\}@eee.hku.hk
}

\begin{abstract}
Terahertz time-domain spectroscopy (THz-TDS) is an emerging modality for biomedical imaging. It is non-ionizing and can detect differences between water content and tissue density, but the detectors are rather expensive and the scan time tends to be long. Recently, it has been shown that the compressed sensing theory can lead to a radical re-design of the imaging system with lower detector cost and shorter scan time, in exchange for computation in the image reconstruction. We show in this paper that it is in fact possible to make use of the multi-frequency nature of the terahertz pulse to achieve hyperspectral reconstruction. Through effective use of the spatial sparsity, spectroscopic phase information, and correlations across the hyperspectral bands, our method can significantly improve the reconstructed image quality. This is demonstrated through using a set of experimental $\mathrm{THz}$ data captured in a single-pixel terahertz system.
\end{abstract}

\section{INTRODUCTION}

In recent years, terahertz $(\mathrm{THz})$ imaging and spectroscopy have been successfully applied to a wide range of applications in medicine and biology, such as tissue diagnosis, breast tumor investigation, illicit drug detection, etc. [1][5]. Among these possible applications, the $\mathrm{THz}$ time-domain spectroscopy (THz-TDS) has demonstrated to be a powerful tool for detecting and characterizing biological material by its response to electromagnetic radiation in the far infrared spectral range [2], [4]. For example, THz-TDS allows us to measure the amplitude and phase of the transient electric field rather than the intensity of the $\mathrm{THz}$ radiation. However, most existing THz-TDS systems suffer from slow acquisition rate because of their raster-scanning mode [4], [6], [7]. To date, the fastest raster-scanning time-domain $\mathrm{THz}$ imaging system needs as long as 6 minutes to scan a $100 \mathrm{~mm}^{2}$ area at $0.25 \mathrm{~mm}$ resolution (equivalently, a $400 \times 400$ pixel image) [7].

Recently, to meet the requirements of practical, time-crucial applications, Chan et al. [8] design a single-pixel THz imaging setup following the compressed sensing (CS) theory [9], [10]. The system schematic is illustrated in Fig. 1(a). The singlepixel $\mathrm{THz}$ receiver samples the object that has been spatially modulated with a random pattern, then one measurement is obtained. The number of measurements depends on how many random patterns are used. From these data, one needs to perform a signal reconstruction step to get the image. The CS theory suggests the conditions that need to be fulfilled to allow nearly perfect reconstruction with a much smaller number of measurements. However, in reality, these conditions

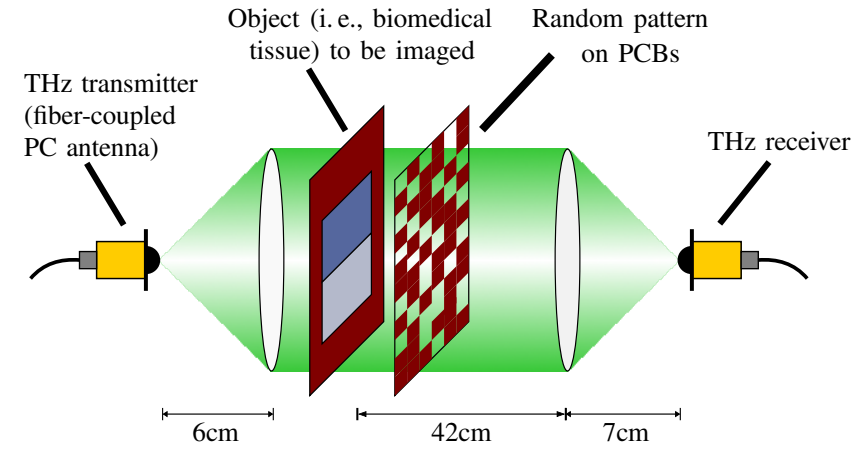

(a)

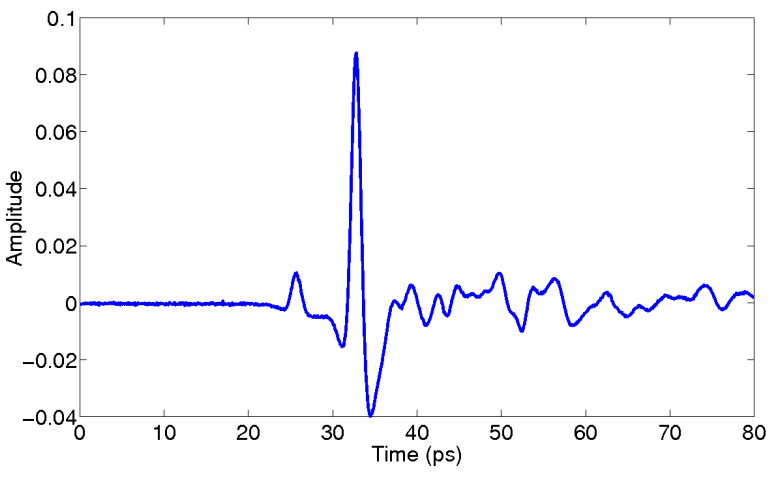

(b)

Fig. 1. (a) Schematic diagram of the single-pixel, pulsed $\mathrm{THz}$ imaging system in [8]. (b) A temporal $\mathrm{THz}$ pulse acquired from such a $\mathrm{THz}$ system.

may not be fully satisfied, and the reconstruction quality may be unsatisfactory. We note, however, that THz-TDS offers two important pieces of information that is not commonly found in other modalities: (i) the $\mathrm{THz}$ pulse (as shown in Fig. 1(b)) contains information at multiple frequencies, and therefore has hyperspectral information [11]; and (ii) at each frequency, the signal is complex, i.e., it has both magnitude and phase information [8], [12]. Thus, in this paper we present a hyperspectral image reconstruction algorithm that takes the spectroscopic phase information and hyperspectral information carried by the $\mathrm{THz}$ pulse into account to deliver better image quality. 


\section{Methodology}

\section{A. Compressed Sensing Reconstruction}

The single-pixel pulsed THz system in Fig. 1 implements a compressed sensing process. Mathematically, at a particular spectral frequency,

$$
\mathbf{y}=\Psi \mathbf{x},
$$

where $\mathbf{y} \in \mathcal{C}^{M}$ is a column vector of measurements and $\mathbf{x}$ represents a $N \times N$ complex-valued image ordered in an $N^{2} \times 1$ vector, sampled by the measurement matrix $\Psi \in$ $\mathcal{R}^{M \times N^{2}}$. Since the imaging scheme requires much fewer measurements (i. e., $M \ll N^{2}$ ), we cannot restore the original signals directly from the observed measurements. However, a very good approximation to the original data can be obtained by solving the following optimization problem [9], [10]

$$
\text { minimize }\|\mathbf{x}\|_{1} \quad \text { subject to }\|\Psi \mathbf{x}-\mathbf{y}\|_{2} \leq \epsilon,
$$

with $\epsilon$ the tolerance to be defined. To solve this, several methods have been proposed, such as the SPGL1 algorithm [13] (which is used for reconstruction in [8]). However, the reconstruction quality is often not satisfactory. On the other hand, we may note that in the optimization problem in Eq. (2), aside from sparsity of the amplitude intensity, any other prior information has not been exploited.

The $\mathrm{THz}$ imaging system in Fig. 1 is a transmission-type spectroscopy. We assume that the object is isotropic, piecewise homogeneous, and has uniform thickness. In this case, one additional piece of prior knowledge about the original data is that the smooth regions in the spatial distribution of amplitude should be the same as those in the spatial distribution of phase. Mathematically, let $x_{1}=A_{1} e^{j \phi_{1}}$ and $x_{2}=A_{2} e^{j \phi_{2}}$ be the complex intensities of two different pixels. If these two pixels are in the same homogeneous region, then $A_{1}=A_{2}$ and $\phi_{1}=\phi_{2}$. Accordingly, we can conclude that the firstorder difference of the complex intensities in a homogeneous region is zero. Therefore, if we define the total variation of the complex data as

$$
\|\mathbf{x}\|_{\mathrm{TV}} \triangleq \sum_{i}\left|\Delta_{i}^{h} \mathbf{x}\right|+\left|\Delta_{i}^{v} \mathbf{x}\right|
$$

with $\Delta_{i}^{h}$ and $\Delta_{i}^{v}$ as linear operators corresponding to, respectively, horizontal and vertical first-order differences at pixel $i$, then minimizing Eq. (3) will not only be a more appropriate choice for sparse reconstruction, but also lead to a solution closer to the reality.

Furthermore, in our recent work [14], we show that the reconstruction can be significantly improved by considering the phase information provided by pulsed $\mathrm{THz}$ spectroscopies. Let $\phi\left(x_{i}\right)$ and $\overline{\phi\left(x_{i}\right)}$ be the phase value of the $i$-th pixel and the mean value of a neighborhood with the $i$-th pixel as the center, respectively. The smoothness constraint on the phase image is defined as

$$
\|\phi(\mathbf{x})-\overline{\phi(\mathbf{x})}\|_{2}=\left(\sum_{i=1}^{N^{2}}\left[\phi\left(x_{i}\right)-\overline{\phi\left(x_{i}\right)}\right]^{2}\right)^{1 / 2} \leq \sigma
$$

with

$$
\phi\left(x_{i}\right)=\left\{\begin{array}{ll}
-j \log \frac{x_{i}}{\left|x_{i}\right|} & \text { if }\left|x_{i}\right| \geq T \\
0 & \text { otherwise }
\end{array}\right\} \in[-\pi, \pi) .
$$

Here $T$ refers to a given threshold for separating the regions containing signal and noise only. If we just consider the case at a single spectral band, the reconstruction algorithm for the CS THz-TDS can be interpreted as an optimization given by

$$
\begin{aligned}
\operatorname{minimize} & \|\mathbf{x}\|_{\mathrm{TV}} \\
\text { subject to } & \|\Psi \mathbf{x}-\mathbf{y}\|_{2} \leq \epsilon \\
& \|\phi(\mathbf{x})-\overline{\phi(\mathbf{x})}\|_{2} \leq \sigma
\end{aligned}
$$

or, equivalently, by the following criterion

$$
\hat{\mathbf{x}}=\underset{\mathbf{x}}{\arg \min } \frac{1}{2}\|\Psi \mathbf{x}-\mathbf{y}\|_{2}^{2}+\lambda\|\mathbf{x}\|_{\mathrm{TV}}+\mu\|\phi(\mathbf{x})-\overline{\phi(\mathbf{x})}\|_{2} .
$$

\section{B. Multiscale Intensity Estimation}

While we have shown that the above single-band method can improve the reconstructed image quality [14], there is still room for further improvement. In particular, THz-TDS provides hyperspectral information about the test object at the THz frequency range, which has not been taken into account in any THz-TDS reconstruction scheme to the best of our knowledge. Information across different spectral bands has high correlation, and that can be used, for example, in [15] where a multiscale photon-limited hyperspectral image reconstruction method is proposed to estimate the true hyperspectral images from the observations corrupted by Poisson noise. Here, we extend this multiscale intensity estimation method to our THzTDS case.

For the hyperspectral images, there is a key feature that the spatial boundaries and singularities at each spectral band are located at the same positions, even when the contrast or perceptibility is very low at some bands [15]. This is also true in THz-TDS. Since the data acquired are complex, we now consider the amplitude and phase separately. We respectively perform recursive dyadic partitioning (RDP) in the amplitude and phase domain. The RDP process on an image produces the quad-tree representation of the image by recursively decomposing any part of an existing partition into dyadic squares which means to replace a square by four similar squares of half the size [16]. Since the partition defined by the RDP is not unique, we use a maximum penalized likelihood estimation to select the optimal partition $\hat{\mathcal{P}}$ which provides the best fit to the observations from the space of possible partitions $\Sigma_{\mathcal{P}}$. Each of the terminal squares of this data-adaptive RDP $\hat{\mathcal{P}}$ corresponds to a spatially homogeneous region. Let $\mathbf{f} \triangleq \mathbf{b} \odot e^{j \boldsymbol{\theta}}$ denote the degraded hyperspectral observations of size $N \times N \times K$, where $e$ is the Euler's number, $\odot$ represents element-by-element multiplication and $K$ is the number of spectral bands. The optimal hyperspectral 


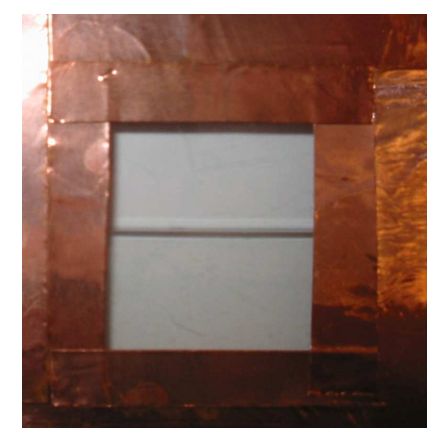

(a)

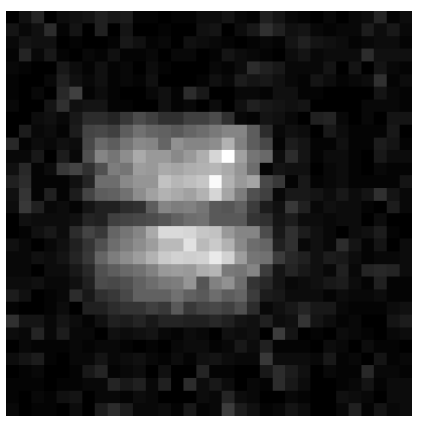

(b)

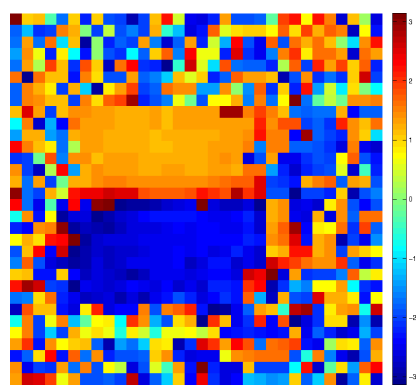

(e)

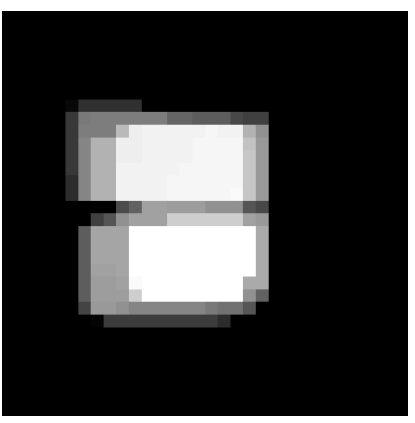

(c)

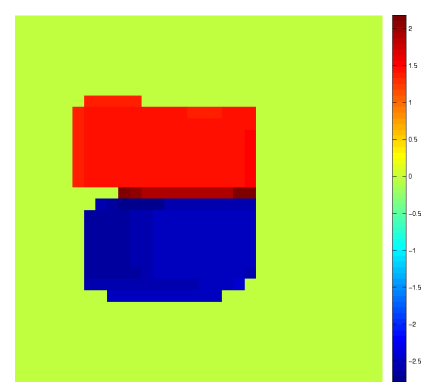

(f)

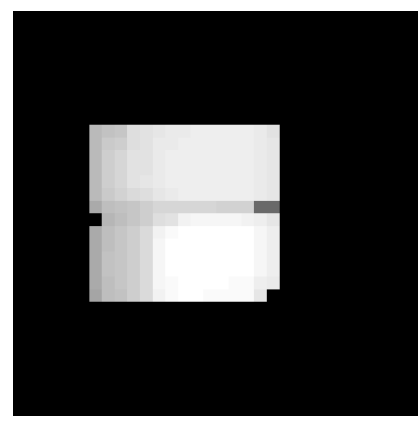

(d)

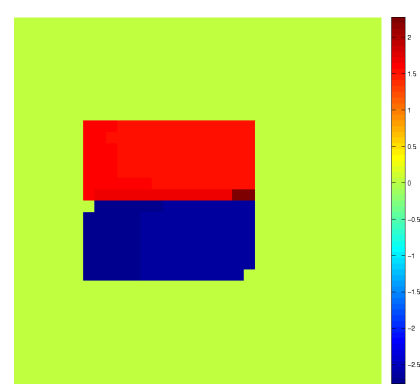

(g)

Fig. 2. Hyperspectral reconstruction results with a $600 \times 16$ practical hyperspectral THz data cube. (a) The rectangular object mask. (b) and (e) The amplitude and phase obtained by applying SPGL1 algorithm [13] at $0.1 \mathrm{THz}$. (c) and (f) The amplitude and phase reconstructed by using the method presented in Section II-A at $0.1 \mathrm{THz}$. (d) and (h) The amplitude and phase obtained by performing the proposed hyperspectral algorithm, displayed at $0.1 \mathrm{THz}$.

estimation of $\mathbf{x} \triangleq \mathbf{a} \odot e^{j \phi} \in \mathcal{C}^{N \times N \times K}$ is then calculated by

$$
\begin{aligned}
\hat{\mathbf{a}}^{\left(c_{\mathbf{a}}\right)} & =\underset{\mathbf{a}^{\left(c_{\mathbf{a}}\right)}}{\arg \min } L_{\mathbf{a}}^{\left(c_{\mathbf{a}}\right)} \\
& =\underset{\mathbf{a}^{\left(c_{\mathbf{a}}\right)}}{\arg \min }\left\{-\log p\left(\mathbf{b}^{\left(c_{\mathbf{a}}\right)} \mid \mathbf{a}^{\left(c_{\mathbf{a}}\right)}\right)\right\}, \\
\hat{\mathcal{P}}_{\mathbf{a}} & =\underset{\mathcal{P}_{\mathbf{a}}}{\arg \min }\left\{\sum_{c_{\mathbf{a}} \in \mathcal{P}_{\mathbf{a}}} L_{\mathbf{a}}^{\left(c_{\mathbf{a}}\right)}+\eta_{\mathbf{a}}\left(\mathcal{P}_{\mathbf{a}}\right)\right\}, \\
\hat{\mathbf{a}} & =\left\{\sum_{c_{\mathbf{a}} \in \hat{\mathcal{P}}_{\mathbf{a}}} \hat{\mathbf{a}}^{\left(c_{\mathbf{a}}\right)}\right\},
\end{aligned}
$$

and

$$
\begin{aligned}
\hat{\phi}^{\left(c_{\phi}\right)} & =\underset{\phi^{\left(c_{\phi}\right)}}{\arg \min } L_{\phi}^{\left(c_{\phi}\right)} \\
& =\underset{\phi^{\left(c_{\phi}\right)}}{\arg \min }\left\{-\log p\left(\boldsymbol{\theta}^{\left(c_{\phi}\right)} \mid \boldsymbol{\phi}^{\left(c_{\phi}\right)}\right)\right\}, \\
\hat{\mathcal{P}}_{\phi} & =\underset{\mathcal{P}_{\phi}}{\arg \min }\left\{\sum_{c_{\phi} \in \mathcal{P}_{\phi}} L_{\phi}^{\left(c_{\phi}\right)}+\eta_{\phi}\left(\mathcal{P}_{\phi}\right)\right\}, \\
\hat{\boldsymbol{\phi}} & =\left\{\sum_{c_{\phi} \in \hat{\mathcal{P}}_{\phi}} \hat{\phi}^{\left(c_{\phi}\right)}\right\} .
\end{aligned}
$$

Here, $p\left(\mathbf{b}^{\left(c_{\mathbf{a}}\right)} \mid \mathbf{a}^{\left(c_{\mathbf{a}}\right)}\right)$ and $p\left(\boldsymbol{\theta}^{\left(c_{\boldsymbol{\phi}}\right)} \mid \boldsymbol{\phi}^{\left(c_{\boldsymbol{\phi}}\right)}\right)$ refer to the likelihood of observing $\mathbf{b}^{\left(c_{\mathbf{a}}\right)}$ and $\boldsymbol{\theta}^{\left(c_{\boldsymbol{\phi}}\right)}$ given the amplitude estimate $\mathbf{a}^{\left(c_{\mathbf{a}}\right)}$ and the phase estimate $\phi^{\left(c_{\phi}\right)}$ in each partition cell $c_{\mathbf{a}} \in \mathcal{P}_{\mathbf{a}}$ and $c_{\boldsymbol{\phi}} \in \mathcal{P}_{\boldsymbol{\phi}}$. They are directly related to the noise distributions in the THz system. The $\eta_{\mathbf{a}}\left(\mathcal{P}_{\mathbf{a}}\right)$ and $\eta_{\phi}\left(\mathcal{P}_{\phi}\right)$ are penalties assigned to the amplitude and phase for encouraging spatial smoothing [17].

\section{Hyperspectral Reconstruction}

Incorporating the sparse reconstruction mentioned in Section II-A with the multiscale estimation technique, we can devise a hyperspectral reconstruction method for the $\mathrm{THz}-$ TDS. It consists of two alternating steps:

Step 1: Let $\hat{\mathbf{x}}^{(t)}$ be the input of the $t$-th iteration. Obtain the solution $\mathbf{f}^{(t)}$ of Eq. (7) across all the observed spectral bands.

Step 2: Considering $\hat{\mathbf{f}}^{(t)}$ as the observations, deal with the amplitude and phase parts separately and get the respective optimal estimators $\hat{\mathbf{a}}^{(t+1)}$ and $\hat{\boldsymbol{\phi}}^{(t+1)}$ according to Eqs. (8-9), then

$$
\hat{\mathbf{x}}^{(t+1)}=\hat{\mathbf{a}}^{(t+1)} \odot e^{\hat{\boldsymbol{\phi}}^{(t+1)}} .
$$

These two steps are executed repeatedly, and the algorithm terminates when

$$
\frac{\left\|\hat{\mathbf{x}}^{(t+1)}-\hat{\mathbf{x}}^{(t)}\right\|_{1}^{2}}{\left\|\hat{\mathbf{x}}^{(t)}\right\|_{1}^{2}}
$$

is less than a given value.

\section{EXPERIMENTAL RESULTS}

In this section, we demonstrate the effectiveness of our proposed hyperspectral reconstruction method on a set of hyperspectral $\mathrm{THz}$ data acquired through an experimental system at Rice University. The test object is a rectangular hole embedded in an opaque screen, filled with two transparent plastic plates of different thickness (see Fig. 2(a)). The reason to choose this kind of object is that it contains different types 
of materials and has features on a relatively small length scale, which mimics the nature of some biomedical specimens.

Our goal in this experiment is to reconstruct the amplitude and phase intensities of size $32 \times 32$ at each observed spectral band with only 600 measurements. Since each measurement obtained from the $\mathrm{THz}$ system is actually a whole pulse signal containing frequency information across the $\mathrm{THz}$ frequency range, we sample the measurements at 16 spectral bands uniformly distributed over the frequency range between $0.1 \mathrm{THz}$ and $0.2 \mathrm{THz}$. Mathematically, we seek to obtain a good $32 \times 32 \times 16$ estimation with $600 \times 16$ measurements.

Figs. 2(b) and (e) show the amplitude and phase obtained by applying the SPGL1 algorithm [13] at $0.1 \mathrm{THz}$. The reconstructed amplitude and phase by using the method presented in Section II-A at the same frequency are shown in Figs. 2(c) and (f). The latter performs much better than SPGL1. However, this single spectral band method fails to preserve some fine edges, and its results can be further improved. Now let us compare our proposed hyperspectral algorithm, shown in Figs. 2(d) and $(\mathrm{g})$, with those two single-band reconstruction approaches. The reconstruction quality is visually the best on both the amplitude and phase images, i.e., sharper edges with less noticeable artifacts. Furthermore, the estimates corresponding to our hyperspectral method is closer to the reality which is indicated in Section II-A.

\section{CONCLUSION}

In this paper, we present an effective hyperspectral reconstruction algorithm for biomedical imaging with $\mathrm{THz}-$ TDS. The key feature of our algorithm is that it effectively employs spatial sparsity, spectroscopic phase information and correlations across the hyperspectral bands to improve the reconstruction quality, despite the limited number of measurements collected. Through the experiments on practical hyperspectral $\mathrm{THz}$ data, our proposed method shows good performance in preserving edges and alleviating artifacts in both amplitude and phase domain. Although in this paper we only discuss THz-TDS in the transmission mode, our method can be readily extended to other modes of $\mathrm{THz}$ spectroscopy, e. g., reflection-type THz-TDS.

\section{ACKNOWLEDGMENT}

This work was supported in part by the Research Grants Council of the Hong Kong Special Administrative Region, China under Projects HKU 713906 and 713408. We acknowledge the help of Wai Lam (William) Chan and Daniel M. Mittleman of Rice University in providing the experimental data.

\section{REFERENCES}

[1] E. Pickwell and V. P. Wallace, "Biomedical applications of terahertz technology," Journal of Physics D: Applied Physics, vol. 39, no. 17, pp. R301-R310, 2006.

[2] G. Png, R. Flook, B.-H. Ng, and D. Abbott, "Terahertz spectroscopy of snap-frozen human brain tissue: an initial study," Electronics Letters, vol. 45, no. 7, pp. 343-345, 2009.

[3] A. J. Fitzgerald, V. P. Wallace, M. Jimenez-Linan, L. Bobrow, R. J. Pye, A. D. Purushotham, and D. D. Arnone, "Terahertz pulsed imaging of human breast tumors," Radiology, vol. 239, no. 2, pp. 533-540, 2006.
[4] K. Kawase, Y. Ogawa, Y. Watanabe, and H. Inoue, "Non-destructive terahertz imaging of illicit drugs using spectral fingerprints," Optics Express, vol. 11, no. 20, pp. 2549-2554, 2003.

[5] A. J. Fitzgerald, E. Berry, N. N. Zinovev, G. C. Walker, M. A. Smith, and J. M. Chamberlain, "An introduction to medical imaging with coherent terahertz frequency radiation," Physics in Medicine and Biology, vol. 47, no. 7, pp. R67-R84, 2002.

[6] N. Karpowicz, H. Zhong, C. Zhang, K.-I. Lin, J.-S. Hwang, J. Xu, and X.-C. Zhang, "Compact continuous-wave subterahertz system for inspection applications," Applied Physics Letters, vol. 86, no. 5, p. 054105, 2005.

[7] D. Zimdars, "High speed terahertz reflection imaging," Proc. SPIE, vol. 5692, pp. 255-259, 2005.

[8] W. L. Chan, K. Charan, D. Takhar, K. F. Kelly, R. G. Baraniuk, and D. M. Mittleman, "A single-pixel terahertz imaging system based on compressed sensing," Applied Physics Letters, vol. 93, no. 12, p. 121105, 2008.

[9] E. J. Candès, J. Romberg, and T. Tao, "Robust uncertainty principles: exact signal reconstruction from highly incomplete frequency information," IEEE Transactions on Information Theory, vol. 52, no. 2, pp. 489-509, 2006.

[10] D. L. Donoho, "Compressed sensing," IEEE Transactions on Information Theory, vol. 52, no. 4, pp. 1289-1306, 2006.

[11] M. C. Kemp, A. Glauser, and C. Baker, "Recent developments in people screening using terahertz technology: seeing the world through terahertz eyes," Proc. SPIE, vol. 6212, p. 62120T, 2006.

[12] W. L. Chan, J. Deibel, and D. M. Mittleman, "Imaging with terahertz radiation," Reports on Progress in Physics, vol. 70, no. 8, pp. 325-1379, 2007.

[13] E. van den Berg and M. P. Friedlander, "Probing the pareto frontier for basis pursuit solutions," SIAM Journal on Scientific Computing, vol. 31, no. 2, pp. 890-912, 2008.

[14] Z. Xu, W. L. Chan, D. M. Mittleman, and E. Y. Lam, "Sparse reconstruction of complex signals in compressed sensing terahertz imaging," in Signal Recovery and Synthesis (SRS). Optical Society of America, 2009, p. STuA4.

[15] K. Krishnamurthy and R. M. Willett, "Multiscale reconstruction of photon-limited hyperspectral data," in IEEE/SP 14th Workshop on Statistical Signal Processing, 2007, pp. 596-600.

[16] D. L. Donoho and X. Huo, "Beamlets and multiscale image analysis," in Multiscale and Multiresolution Methods: Theory and Applications, ser Lecture Notes in Computational Science and Engineering, T. J. Barth, T. Chan, and R. Haimes, Eds. Springer, 2001, pp. 149-196.

[17] R. M. Willett and R. D. Nowak, "Multiscale poisson intensity and density estimation," IEEE Transactions on Information Theory, vol. 53, no. 9, pp. 3171-3187, 2007. 